\title{
External visual interface for a Nikon 6D autocollimator
}

\author{
Guillermo Bergues \\ Guillermo Ames \\ Luis Canali \\ CIII at UTN-FRC \\ Córdoba, Argentina \\ gbergues@cbasicas.frc.utn.edu.ar
}

\author{
Clemar Schurrer \\ CEMETRO at UTN-FRC \\ Córdoba, Argentina \\ cschurrer@cbasicas.frc.utn.edu.ar
}

\author{
Ana Georgina Flesia \\ Famaf-UNC \\ Conicet at UTN-FRC \\ Córdoba, Argentina \\ flesia@mate.uncor.edu
}

\begin{abstract}
The goal of this paper is to describe the potential of a visual interface applied to a Nikon $6 \mathrm{~B} / 6 \mathrm{D}$ autocollimator in order to replace human operator within a "laboratory grade" measurement. The optical interface implemented consists of a Basler HD camera attached to the autocollimator's eyepiece, camera positioning devices, and a proprietary digital image processing package, built around Matlab environment. The whole system is used to analyze the reticle image to detect both the scale and the measuring crosshair lines at sub-pixel level. The system performance was compared in a controlled experiment against an electronic level with internationally traceable certification. The resolution of the pitch angles obtained was 10 times better than that originally obtained with the instrument. This example shows that the value of 0.02 pixels for the uncertainty associated with sub-pixel position of reticle lines is realistic, allowing for continue working on a robust interface for all angle parameters that can be measured using autocollimators.
\end{abstract}

\section{INTRODUCTION}

An autocollimator is an optical instrument that measures angles with no physical contact and high accuracy. This device is very useful when measuring parallelism, perpendicularity, straightness, and pitch and yaw angles. These above mentioned parameters are of vital importance in the metrological evaluation of Coordinate Measuring Machines and Machine Tools in production plants or metrology laboratories. They are also an indispensable tool for the characterization of the topography of surfaces on ultra precise mirrors for X-ray optics, [1]-[2].

To perform a measurement with an autocollimator the operator places one of his eyes against the instrument's eyepiece and observes the displacement of the crosshair lines on the measurement reticle. This kind of procedure induces systematic and random errors due to the existence of parallax, inherent to this method, and defects of the observer's eye.

This paper presents the implementation of an external (i.e. no tampering of the instrument required) visual interface to a Nikon 6B/6D standard autocollimator, comprising a digital camera, a computer for processing the camera images, and the image processing package built around Matlab / Octave environment. This system reduces the previous errors.

This interface allows for extend the resolution of the instrument, since the camera image has much more information available than that the operator can resolve inspecting the autocollimator measurement reticle. This information is processed so as to determine the position of the center of a line with subpixel resolution.

The location of curves within a picture with sub-pixel resolution is of high interest in very different fields, such as glass width estimation, [3]; high temperature specimen contour estimation, [4]; among others.

In Section II we describe the operational principles of the autocollimator, and we introduce the basic concepts employed when measuring using these instruments. Finally, we describe how we proceeded to make a measurement with the visual interface. In Section III we discuss the results of segmentation and detection algorithms that were developed to reproduce the work of the operator during the measurement. Conclusions and future work are detailed in the final section.

\section{EXPERIMENTAL}

\section{A. Autocollimator}

An autocollimator (Fig. 1) is an optic instrument used for measuring small angular displacements (at the seconds of arc level).

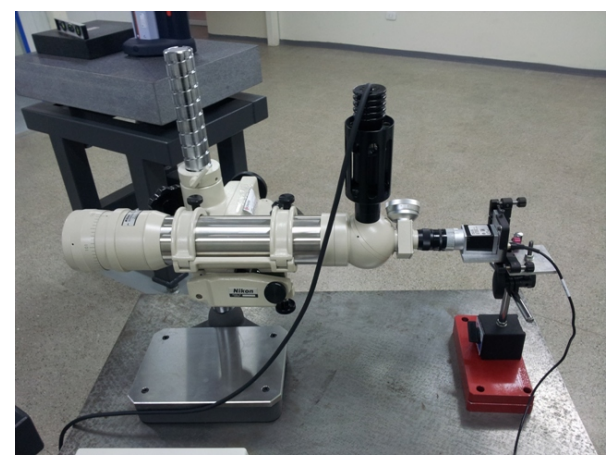

Fig. 1: Nikon 6D Autocollimator and Basler Camera, located in front of the autocollimator's eyepiece.

It has the capability of performing measurements without making contact with the measured object. This is a very useful feature within an assembly line or in a reference lab, where instruments and ancillary devices must be manipulated delicately. 
1) Working Principle: To perform a measurement, the autocollimator works together with a reflecting surface $E$, whose distance to the autocollimator has no influence on the measurement.

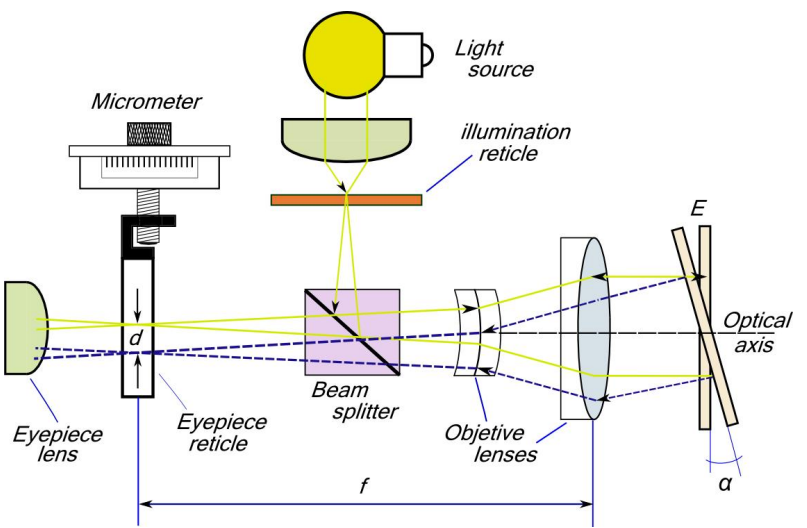

Fig. 2: Autocollimator functioning scheme, where $f$ is the focal distance, $d$ the displacement, and $\alpha$ the measured angle.

The autocollimator projects a collimated light beam parallel to its objective's axis. The light, from the light source, is sent through the illumination reticle and takes the form of crosshair lines, then it is bent through a right angle by the beam splitter that has a semi-transmissive mirror, and becomes a parallel beam as it passes through the objective lenses. After that, it reflects on the mirror $E$ and travels back to its origin, where its angular deviation with respect to the instrument's optical axis is measured. If $E$ is perpendicular to the instrument's optical axis, the projected light beam (yellow lines in Fig. 2) is reflected by the mirror $E$ towards its origin and in the eyepiece reticle no displacement $d$ is observed. On the other hand, if $E$ is not perpendicular with respect to the instrument's optical axis, the reflected beam (blue dot lines in Fig. 2) is shifted with respect to the calibrated measuring scale in such a way that a displacement "d" on the scale becomes the inclination angle $\alpha$. The micrometer allows for measure $\alpha$ with a resolution $R=0.5$ seconds of arc in our case. The calibration ratio is given by "(1),":

$$
\tan (2 \cdot \alpha)=\frac{d}{f},
$$

where $f$ is the focal distance.

\section{B. Measurement with Visual Interface}

In Fig. 3a, an image of the autocollimator's reticle scale can be seen. There, the instrument scale and the crosshair lines indicating pitch $\left(B_{y}\right)$ and yaw $\left(B_{x}\right)$ angles can be distinguished, and, in Fig. $3 \mathrm{~b}$ is present the scheme and variables that are important to calculate in order to obtain the measurement with the visual interface.

Using Fig. $3 b$ to explain the process, the procedure to perform an automated measurement is:

1) Establish the distance $\Delta_{X Y}$ between the center of the divisions of the reticle scale at sub-pixel level. $\Delta_{X Y}$ uncertainty estimation.

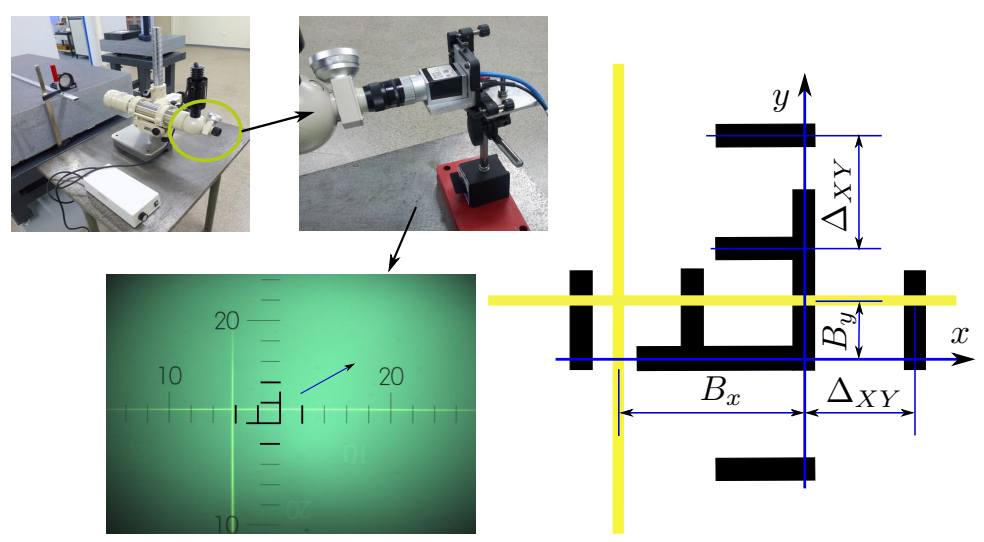

(a) Image of the reticle scale.

(b) Measuring process scheme.

Fig. 3: Correspondence between image and measurement.

2) A coordinate system $(x, y)$ is associated to the image reticle.

3) Identify the crosshair lines at sub-pixel level and measure the distance $B_{y}$ and $B_{x}$ between the center of the lines forming the cross with each axis.

For the Nikon 6D autocollimator, the distance between consecutive divisions of the scale represents 60 seconds of arc $(1 \mathrm{div}=1 \mathrm{~min})$. Once the value $\Delta_{X Y}$ of pixels/division is obtained, the observed angle (e.g., pitch) in seconds of arc can be obtained using:

$$
\alpha_{y}=60 \cdot \frac{B_{y}}{\Delta_{X Y}}
$$

1) Image-capturing process: To obtain a precise image, such as those shown in Fig. 7a and Fig. 7b, it is necessary to:

1) Focus and position the camera in such way that the incoming light to the telescope is centered in the image: Determining when the camera is in focus is a delicate point [5],[6], since there is a trade off between picture sharpness and number of pixels to determine the position of the lines at sub-pixel level. Also, the autocollimator's telescope is formed by a set of lenses that reflect the outside light through the eyepiece area (where the final image is formed), generating radial lighting saturated zones in the image center and dark areas at the image contour (see Fig. 4). If this incoming light has an offset and it is not centered in the reticle, the final image will have a wrong structure. Because of that, the image processing software will compute with errors. Therefore, system calibration consists in determining the autocollimator's zero, moving the camera until the axes of the autocollimator's objective and camera lenses are aligned and, the measurement reticle is in focus. This is done once before measuring, it is used a calibration software that measured the image intensity matrix and then it is obtained the best camera position. We studied the best focus 
position and sharpness using a set of different lenses with a separate set of images, and fix it for all the experiments.

$I N C O R R E C T=O F F S E T$

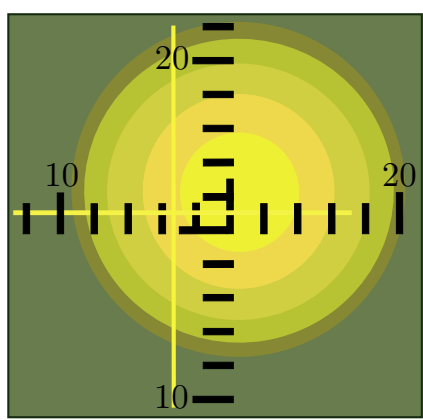

Fig. 4: Scheme of how is has to be seen the incoming light in the picture

2) Align the real lines with grid camera pixels: The software is created so that it needs the image lines to fall into the square pixels of the CCD. It is performed a spatial calibration only with the cross horizontal position as shown in Fig. 5: the pixel highs, had to be the same $h_{1}=h_{2}$.
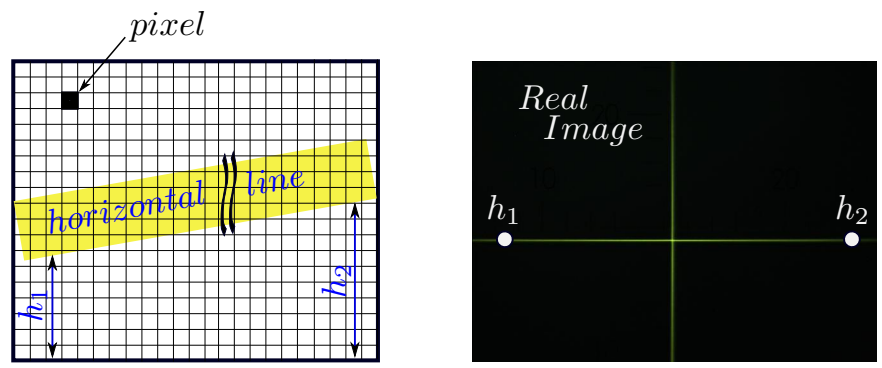

Fig. 5: Scheme of how is has to be corrected the alignment of the lines respect the grid camera pixels

3) Separate the reticle from the crosshair lines: The Nikon 6D is a dark field autocollimator, optimized in such a way so that the operator can place the crosshair lines at the center of the reticle looking through the instrument's eyepiece. To this purpose, the darker the ambient, the better the precision attained in placing the crosshair lines. However, this situation is not optimal to obtain a good picture of the reticle scale. A picture where both the scale and the crosshair lines can be easily seen leads to errors in the determination of the position of the lines that form them, and these errors are unacceptable in the context of a "laboratory grade" measurement.

However, the observation of the scale and the crosshair lines can be made separately, with no movements on the positioning system of the camera, simply changing ambient light. As images do not suffer displacements by changes in the illumination, it is possible to gather different images of the crosshair lines and the scale for each position of the mirror.
Thus, after performing the mirror alignment and camera placement, images of the reticle (see Fig.7a) were taken with the lights on in order to maximize the contrast between the clear, bright background and the dark lines of the reticle scale. This set of images was used only once, to compute the number of pixels/division $\Delta_{x y}$ of the system, completing the calibration of the system.

To perform the measurements, seeing the displacement of the crosshair lines, the lights in the room were turned off, capturing only images with the crosshair lines (see Fig. 7b) since the background (and the reticle scale) are dark, and the cross itself is bright.

\section{Measuring Experiment}

To validate the developed procedure with the visual interface, a controlled experience was designed: In each experiment angles were generated using a micrometric screw, and measured simultaneously using the system under test (autocollimator + camera + software) and an electronic level. The Mahr electronic level was used as reference. The electronic level is a Mahr Federal EMD-832P-48-W2; Serial Number 2095-06293. This instrument is available within the Laboratory where the experiment is performed (CEMETRO, UTN Córdoba) and it is readily traceable to internationally accepted standards. The electronic level resolution is 0.1 arc seconds and the accuracy is within $2 \%$.

The mirror $E$ and an electronic level $N$ are placed on a bar that can rotate around an axis. Bar rotation is regulated by means of a micrometric screw $T$ placed on one of its ends (see Fig. 6). The working assumption is that the electronic level $N$, for the $k$-th position of screw $T$ generates a reference measurement of a pitch angle, so the results of the experience are to be ordered pairs $\left(X_{k}, \alpha_{k}\right)$, where $X_{k}$ is the level reading and $\alpha_{k}$ is the autocollimator value once the images obtained with the camera are processed.

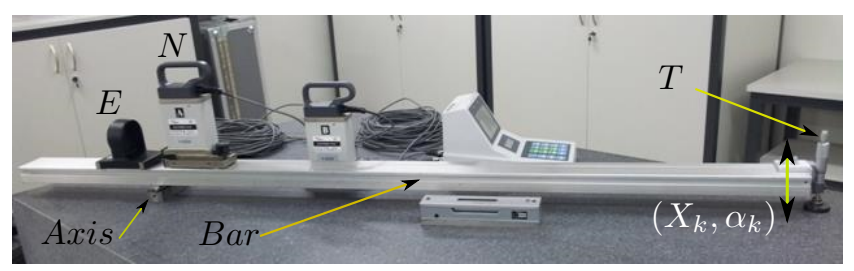

Fig. 6: Experience with electronic level and autocollimator.

\section{Pattern recognition on the captured images}

The digital interface generates two sets of color images: one set corresponds to the reticle scale of the autocollimator reticle (Fig. 7a), and the other to the crosshair lines (Fig. 7b). A total of 500 images of the cross (20 for each position) and 100 for the scale were taken in order to perform the pattern recognition. Both sets of digital images have figures made of sets of straight lines. The position of these lines has to be determined very accurately in order to infer the value of the measured angle. 


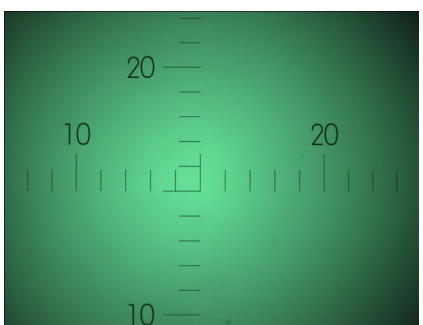

(a) Reticle scale image.

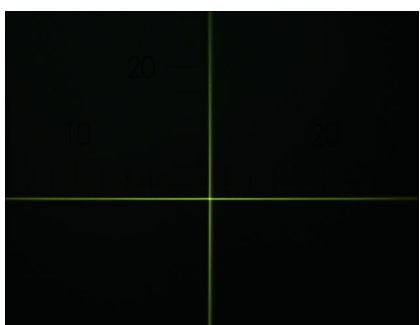

(b) Crosshair line image.
Fig. 7: Image set.

1) Establish the distance $\Delta_{X Y}$ : The first stage on the detection is to model the measurement reticle (eyepiece reticle). Using the algorithm shown in Fig. 8, we obtain the distance between segments.
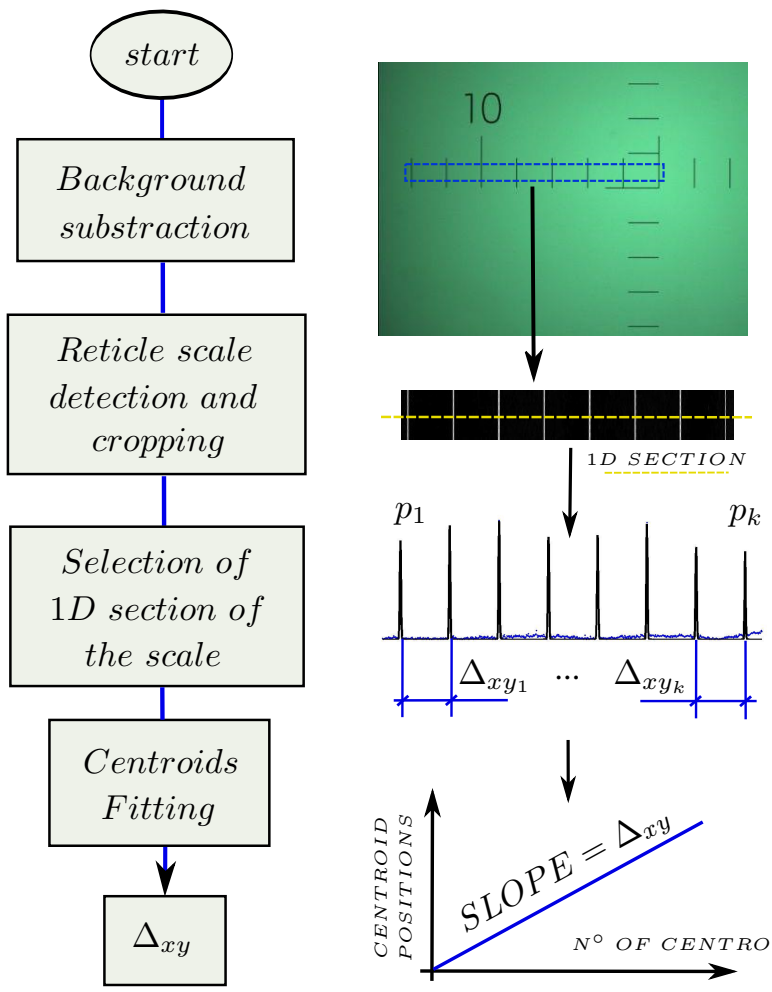

Fig. 8: Flowchart detection of $\Delta_{X Y}$.

We have work using eight vertical and horizontal scale segments taken from the center of the image. This was carried out to minimize optical aberration. A linear fitting to the eight obtained segment centres $p_{k}$ is performed using a simple regression model "(3),".

$$
p_{k}=\Delta_{X Y} * k+\epsilon, \quad k: 1, \ldots, 8, \quad \epsilon \sim N(0, \sigma) .
$$

Estimated sub-pixel values for the autocollimator scale pitch on each axis are coincident within the $95 \%$ confidence interval. Therefore a mean value for scale calibration can be set as:
$\Delta_{X Y}=(97.31 \pm 0.02)$ pixels/division (95\% confidence).

This allows for conclude that scales are linear within an uncertainty margin of 0.02 pixels/div for both axes.

2) Identify the crosshair lines: To perform the detection we propose the algorithm that is explain in Fig. 9.

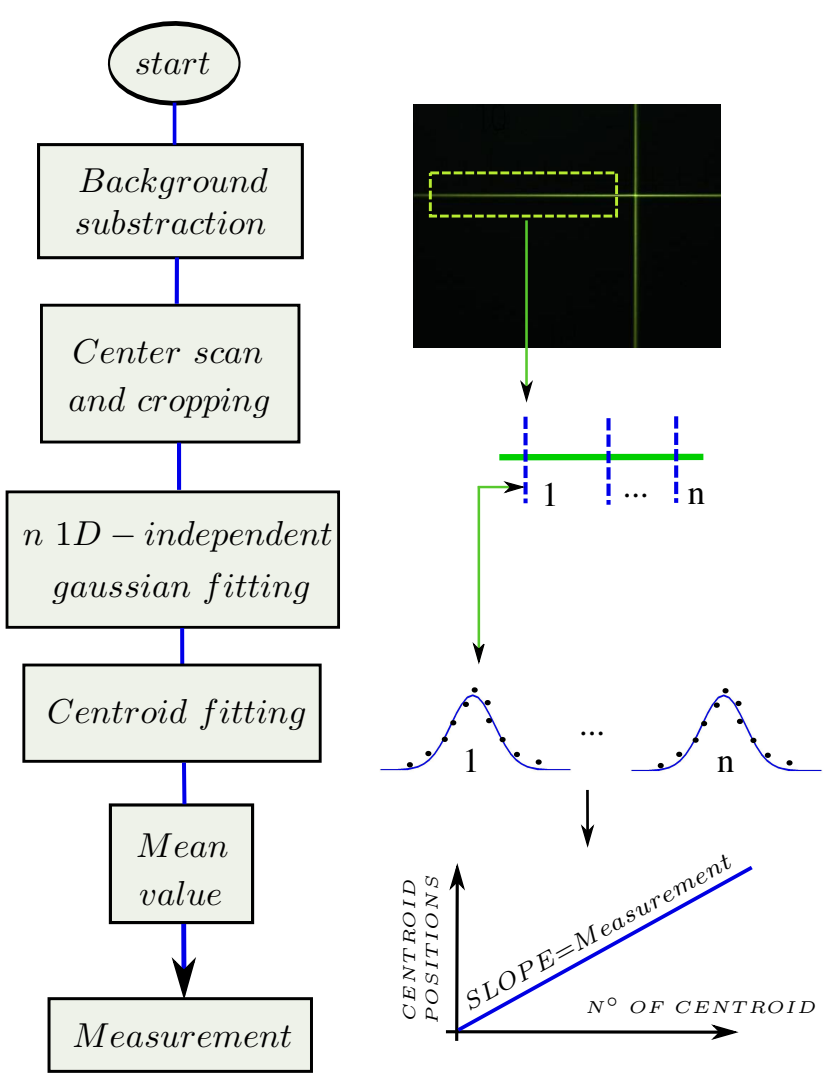

Fig. 9: Flowchart detection crosshair lines position.

The position of the one dimensional straight line is estimated with the centroid of a Gaussian function, as it was carried out with the scale. It is named $B_{k}$.

$\alpha_{k}$ is the relative position with respect to centroid $B_{1}$, corresponding to the reference value (first measurement) of the visual interface and the electronic level. It is converted to arc seconds using "(5),”:

$$
\alpha_{k}=\frac{\left(B_{k}-\left(B_{1}\right)\right) * 60}{\Delta_{X Y}}=\frac{\left(B_{k}-\left(B_{1}\right)\right) * 60}{97.31} .
$$

\section{RESULTS AND DISCUSSION}

A. Comparison of measures performed with the modified autocollimator and the electronic level

The data $\left(X_{k}, \alpha_{k}\right)$ for $k=1 \ldots 25$ were fitted with a linear regression model:

$$
\alpha_{k}=a * X_{k}+b+\epsilon, \quad \epsilon \sim N(0, \sigma)
$$


The slope $a=1.0232 \pm 0.0004$ (95\% confidence) and the intercept $b=0.81 \pm 0.02$ seconds of arc (95\% confidence).

The difference between $(a)$ and unity is near $2 \%$. This value is very close to the electronic level accuracy.

The error in the fitting produced for the calibration of the Electronic Level was disregarded because this research is focused on the new instrument's resolution. This indicates that errors in measurements using the camera plus the autocollimator ensemble are not random in nature.

The discrepancies are define on:

$$
D_{k}=\alpha_{k}-\left(a * X_{k}+b\right)
$$

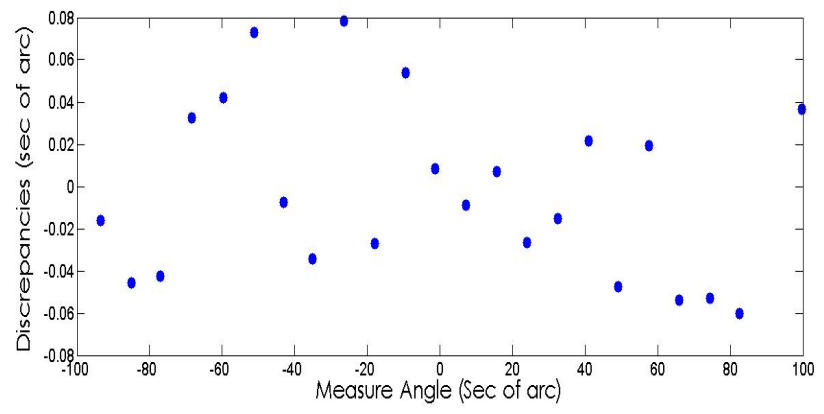

Fig. 10: Plot of the discrepancies $D_{k}$ vs. $X_{k}$.

The mean square value $\left(D_{r m s}\right)$ of discrepancies calculated in this controlled experiment is:

$$
D_{r m s}=\sqrt{\frac{\sum_{k} D_{k}^{2}}{25}}=0.04 "
$$

Therefore it is possible to estimate the resolution gain $(G)$ of the vision system with respect to that of the original instrument $(R=0,5$ ") as:

$$
G=\frac{R}{D_{r m s}}=\frac{0.5}{0.04}=12.5 \sim 10
$$

The results show a tenfold net increase in the instrument measurement resolution when is replaced the operator with an automated procedure using this visual interface.

\section{CONCLUSIONS AND FUTURE WORK}

In this paper the main characteristics of a visual interface are described. The interface comprises a CCD camera and an image processing package custom built around Matlab environment. The interface was set up to improve the resolution of measurements performed with a standard Nikon 6D dark field autocollimator. The results indicate that a $10 \mathrm{x}$ increment in resolution is feasible. The measurements were performed using two set of images obtained independently from each other, namely those of the reticle scale and the crosshair lines used in angle measurement. The procedure to obtain the reticle scale values is carried out just once, since it depends solely on the camera resolution and not on measured values. The key for angle calculation is the use of image sections that were modeled with Gaussian functions. Centroids of these functions allow to determine the position of each line of the scale with sub-pixel resolution.

An improved experimental setup is also needed to generate angles covering 30 minutes of arc, the whole range of the autocollimator. On the other hand it will be necessary to obtain a more accurate reference for the angle values, to calibrate and determine the different contribution to the uncertainty of measurement of angles. Work on sub-pixel modeling should cover, besides those resolution aspects covered partly in this paper, uncertainty issues related to angle calculation, in accordance with ISO-GUM Protocol.

\section{ACKNOWLEDGEMENTS}

This research has been partially supported by Foncyt, Secyt-UNC y Secyt-UTN, under PICT 2008-00291, PID UTN 2012- 25/E170, PID UTN 1406, and PID 2012 05/B504 grants.

\section{REFERENCES}

[1] R. Soufli, M. Fernandez-Perea, S.L. Baker, J.C. Robinson, E.M. Gullikson, P. Heimann, V.V. Yashchuk, W.R. McKinney, W.F. Schlotter, and M. Rowen, "Development and calibration of mirrors and gratings for the soft x-ray materials science beamline at the Linac Coherent Light Source free-electron laser," Appl Opt. , vol. 51, no. 12, pp. 2118-28, Apr 2012.

[2] F. Siewert, J. Buchheim, T. Hoft, S. Fiedler, G. Bourenkov, M. Cianci, R. Signorato, "High resolution slope measuring deflectometry for the characterization of ultra-precise reflective X-ray optics," 56th International Scientific Colloquium, Ilmenau University of Technology, pp. 12-16, September 2011.

[3] J.B. Park, J.G. Lee, M.K. Lee, S.E. Lee,"A glass thickness measuring system using the machine vision method," International Journal of Precision Engineering and Manufacturing, vol. 12, no. 5, pp 769-774, October 2011.

[4] A. Fabijanska, D. Sankowski, "Computer vision system for high temperature measurements of surface properties," Machine Vision and Applications, vol. 20, no. 6, pp. 411-421, 2009.

[5] Goldsmith, N. T. (2011). Deep Focus; A digital image processing technigue to produce improve focal depth in light microscopy. Image Analysis and Stereology, [S.1.], v. 19, n. 3, p. 163-167, may. 2011

[6] Vollath, D.(1988). The influence of the scene parameters and of noise on the behaviour of automatic focusing algorithms. Journal of Microscopy, vol. 151, n. 2 pp. 133-146, Aug. 1988

[7] M. Hagara, P. Kulla, "Edge detection with sub-pixel accuracy based on aproximation of with ERF function," Radioengineering, vol. 20, no. 2, June 2011 .

[8] T. Hermosilla, E. Bermejo, A. Balaguer, L.A. Ruiz,"Non-linear fourthorder image interpolation for subpixel edge detection and localization" Image and Vision Computing, vol. 26, no. 9, pp. 1240-1248, 2008.

[9] A.J. Tabatabai, O.R. Mitchel, "Edge location to subpixel values in digital imagery," IEEE Transactions on Pattern Analysis, Machine Intelligence, vol. 11, no. 12, pp. 1293-1309, 1989. 